\title{
Excitation-wavelength Dependent Fluorescence of Ethyl 5-(4-aminophenyl)-3-amino-2,4-dicyanobenzoate
}

\author{
Marek Józefowicz • Janina R. Heldt
}

Received: 16 April 2010 /Accepted: 16 August 2010/Published online: 27 August 2010

(C) The Author(s) 2010. This article is published with open access at Springerlink.com

\begin{abstract}
The excitation wavelength dependence of the steady-state and time-resolved emission spectra of ethyl 5(4-aminophenyl)-3-amino-2,4-dicyanobenzoate (EAADCy) in tetrahydrofuran (THF) at room temperature has been examined. It is found that the ratio of the fluorescence intensity of the long-wavelength and short-wavelength fluorescence bands strongly depends on the excitation wavelength, whereas the wavelengths of the fluorescence excitation and fluorescence bands maxima are independent on the observation/excitation wavelengths. The dynamic Stokes shift of fluorophore in locally excited $(L E)$ and intramolecular charge transfer (ICT) states has been studied with a time resolution about $30 \mathrm{ps}$. The difference between Stokes shift in the $L E$ and $I C T$ states was attributed to the solvent response to the large photoinduced dipole moment of EAADCy in the fluorescent charge transfer state. On this base we can state that, the relaxation of the polar solvent molecules around the fluorophore was observed.
\end{abstract}

Keywords Ethyl 5-(4-aminophenyl)-3-amino-2,

4-dicyanobenzoate $\cdot$ Donor-acceptor system .

Excitation-wavelength dependent fluorescence

\section{Introduction}

Fluorescence spectroscopy represents an appropriate technique for investigating structural properties of fluorophores in liquid system [1]. The electronic absorption and emission

M. Józefowicz $(\bowtie) \cdot J$. R. Heldt

Institute of Experimental Physics, University of Gdańsk,

ul. Wita Stwosza 57,

80-952 Gdańsk, Poland

e-mail: fizmj@univ.gda.pl spectra of molecules in solution, respectively, give reliable information about solvation effects in the ground and excited states. In general, fluorescence emission of molecules is governed by Kasha's rule which states that fluorescence normally occurs from the lowest vibrational level of the first excited electronic state. It is obvious from this rule that fluorescence should be independent on the excitation wavelengths. On the other hand, it is known for some time, that the fluorescence bands maxima of some system (polar molecules) can shift towards longer wavelengths with an increase in the excitation wavelength [2-15]. This phenomenon is commonly known as red-edge effect [2]. Since this effect is observed only under conditions of restricted mobility, it has been used as a potential tool to estimate the fluorophore (both intrinsic and extrinsic) environment in the organized biological assemblies as membranes, micelles, and proteins [2-15].

It is generally accepted that wavelength-dependent fluorescence involves emission from multiple states or different conformers of the molecule. In a medium where inhomogeneous distribution of species appears, each solute molecule with its own surrounding possesses different absorption and fluorescence band. As a result of this the absorption and emission spectra possess a complex nature i.e., an inhomogeneous broadening of electronic spectra. These conformations of solute molecules interchange rapidly in a fluid medium whereas in a rigid medium the probe molecules are trapped in various geometric configurations.

Ethyl 5-(4-aminophenyl)-3-amino-2,4-dicyanobenzoate (EAADCy, see insert of the Fig. 1) belongs to the fluorophores which show significant red-edge effect [10]. The fluorescence intensity maximum position of the molecule under study in THF glass at $77 \mathrm{~K}$ shows considerable dependence on the excitation wavelengths. The origin of this behavior lies in the variety of solute-solvent interaction (fluorescence centers) in 


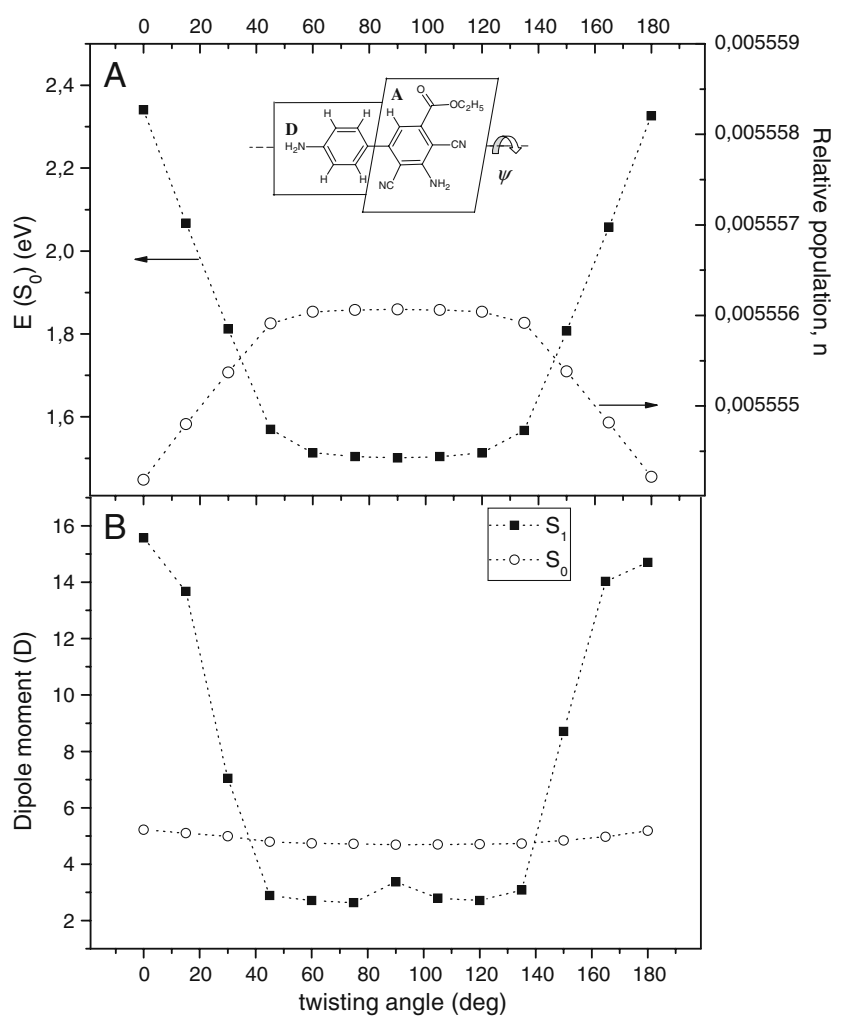

Fig. 1 a Ground-state rotational potential and the corresponding rotamer distribution function $n(\varphi)$, b Dipole moments in the ground and first excited states as a function of the dihedral angle

the ground and excited states. Such assembles of solute molecules excited in a narrow band causes a photoselection of different conformers of EAADCy, which emit own characteristic band.

In the present paper, we are continuing our previous investigation of the spectral inhomogenity of EAADCy. We present results of systematic studies concerning the excitation-wavelength dependent dual fluorescence of EAADCy. Steady-state and time-resolved measurements are made on molecule under study in THF at room temperature. It is found that the ratio of the fluorescence intensity of the long-wavelength and short-wavelength fluorescence bands $\left(I_{e x c}(L W) / I_{e x c}(S W)\right.$ and $\left.I_{e m}(L W) / I_{e m}(S W)\right)$ strongly depends on the observation/excitation wavelength, whereas the wavelengths of the fluorescence excitation and fluorescence bands maxima are independent on observation/ excitation wavelengths.

\section{Theoretical Background}

Recently, several theoretical models of the solvation of molecule in liquid solvent have been developed [1]. Phenomenological description of inhomogeneous broadening of electronic spectra of organic molecules in solution has been given by Nemkovich et al. [11, 12]. They proposed a free energy diagram which describes the dependence of the free energy of the probe-environment centers (solvates) on the strength of the local electric field. According to Nemkovich et al., the ground-state solvate free energy can be written as:

$U_{g}(R)=\frac{\left(R-R_{g}^{e q}\right)^{2}}{2 f}$,

where $R_{g}^{e q}$ is the electric field strength of the equilibrium solvate, $f$ is the factor of the reaction field, which, in a linear approximation, equals to the coefficient of proportionality between the field and ground-state electric dipole moment $\left(\mu_{\mathrm{g}}\right) R_{g}^{e q}=f \mu_{g}$. The free energy of the excited solvates is given by $[11,12]$ :

$U_{e}(R)=\frac{\left(R-R_{e}^{e q}\right)^{2}}{2 f}+h v_{0}+\frac{f}{2}\left(\vec{\mu}_{e}+\vec{\mu}_{g}\right)\left(\vec{\mu}_{e}-\vec{\mu}_{g}\right)$,

where $v_{0}$ is the frequency of the pure $(0-0)$ electronic transition of the free probe molecule, $h$ the Planck constant, $\mu_{e}$ is the dipole moment in the excited state. $R_{e}^{e q}$ is the local electric field in the solvent established by the solute molecules in the thermally equilibrated excited state.

From the above, it is evident that inhomogeneous broadening occurs because different set of solute molecule possesses different solvation shell and different strength of the reaction field $R$. The range of the inhomogeneous broadening, which dictates photoselection of the energetically different species, depends on the values of dipole moments of the dye molecules in the ground and excited states, and on the dielectric properties and structure of a solvent $[11,12]$.

As it was mentioned earlier, the excitation-wavelength dependence of fluorescence band intensity is a result of the existence of energetically different luminescence centerssolute molecules with different shell structure of the nearest solvent molecules (e.g., presence of various solute conformers having different geometries and absorbing at different wavelengths). In this case, it is necesary to use the rotamer distribution function $n(\varphi)$ determined from Boltzmann's law:

$n(\varphi)=e^{\frac{-E(\varphi)-E\left(\varphi_{e q}\right)}{R T}} / \int_{0}^{180} e \frac{-E(\varphi)-E\left(\varphi_{e q}\right)}{R T} d \varphi$.

Excited states processes are usually studied by measurements of the time-resolved emission spectra. In such experiments, light absorption promotes molecules to non-relaxed FranckCondon state, which undergoes fast transition to the relaxed equilibrium singlet state. This dynamic process can be 
observed monitoring the time depending position of maximum of the fluorescence spectrum. The relaxation dynamic between solute and solvent molecules are generally expressed in terms of the correlation function $c(t)$, defined as [16]:

$c(t)=\frac{\widetilde{v}(t)-\widetilde{v}(\infty)}{\widetilde{v}(0)-\widetilde{v}(\infty)}$,

where $\widetilde{v}(t), \widetilde{v}(0)$, and $\widetilde{v}(\infty)$ are the wavenumbers of the fluorescence band maximum at times $t, 0$, and $\infty$. The continuum model of the solution [16] predict an exponential decay of the correlation function, $\tau=\exp \left(-\mathrm{t} / \tau_{L}\right)$, with a longitudinal relaxation $\tau_{L}$ time $\left(\tau_{L}=\tau_{D} \frac{\varepsilon_{\infty}}{\varepsilon_{0}} ; \tau_{D}\right.$ denotes the dielectric relaxation time, $\varepsilon_{0}$ and $\varepsilon_{\infty}$ the static and optical permittivity). It is important to note here that many experiments investigating solvation dynamics have shown that this model does not account fully for the observed changes of $c(t)$.

\section{Experimental Details}

The solvent used in the present study (tetrahydrofuranTHF) was the spectroscopic grade and was used without any additional purification. Absorption, fluorescence excitation and emission spectra at room temperature were recorded using, respectively, a Shimadzu UV-2401 PC spectrophotometer and a Shimadzu RF-5301 spectrofluorometer with $5.0 \mathrm{~nm}$ band-widths in both excitation and emission. Fluorescence measurements were carried out using the triangular cuvette with frontal excitation to minimize the effect of reabsorption. The emission was observed perpendicular to the direction of the exciting beam. Thus the errors due to fluorescence reabsorption were reduced in a way that mathematical corrections were superfluous.

Time-resolved emission spectra of the sample were measured applying streak camera (C4334-01 Hamamatsu) and $2501 \mathrm{~S}$ spectrograph (Bruker Optics). Solid state Nd: YAG laser (PL 2143A/SS EKSPLA) and optical parametric generator (PG 401/SH EKSPLA) were used as an excitation light pulses source [17].

\section{Result and Discussion}

\section{Steady-state Spectra of EAADCy}

To test whether the changes in the absorption, fluorescence excitation and fluorescence spectra originate from the solute conformers possesing different angles between their interatomic planes of the donor and acceptor moieties, a series of conformational semiempirical quantum mechanical calculations were carried out. We found that for EAADCy molecule the ground-state rotational potential is essentially flat (in the range from $60^{\circ}$ to $120^{\circ}$ ) $[18,19]$. Figure 1a shows the calculated ground-state $\left(S_{0}\right)$ rotational potential and the corresponding rotamer distribution function $n(\varphi)$ determined from Boltzmann's low at $298 \mathrm{~K}$, for the molecule under study. According to the Boltzmann's low, in the ground state about $33 \%$ of the all EAADCy molecules possess twist angle between two fragments (donor and acceptor) in the range from $60^{\circ}$ to $120^{\circ}$. Thus, quantum-chemical calculations suggest the presence of various conformers in the ground state which have different geometry and absorb at different wavelengths. In addition, it should be noted that $\varphi_{e q}$ (obtained using quantum-chemical calculations) does not differ from the mean twisting angle $\left\langle\varphi_{n}\right\rangle=\int_{0}^{180} \varphi n(\varphi) d \varphi$, which is convoluted with the distribution function $n(\varphi)$, and equals $\varphi_{e q}=<\varphi_{n}>\cong 90^{\circ}$.

As it was mentioned earlier, the range of inhomogeneous broadening depends on the values of dipole moments of the dye molecules in the ground and excited states. From the quantum-chemical calculations [18, 19] (see Fig. 1b) it is evident that dipole moment of EAADCy in the ground state shows a very week dependence on the dihedral angle, whereas in the first excited state planar molecule possesses five times greater dipole moment than that with perpendicular donor-acceptor arrangement (i.e., $15.6 \mathrm{D}$ versus $3.0 \mathrm{D})$. The large differences between $\mu_{S_{1}}(L E)$ and $\mu_{S_{1}}(I C T)$ suggest existence of molecules possessing different solvation shell and different strength of the reaction field $R$.

Figure $2 \mathrm{a}$ presents the observation-wavelength dependence of fluorescence excitation spectra (maxima position and shape of the spectra) of EAADCy in THF at room temperature. The major observation that can be made suggests the strong dependence of the fluorescence excitation spectrum on the observation wavelength. The long-wavelength fluorescence excitation band of EAADCy are composed of at least two bands, centered at about $360 \mathrm{~nm}$ and $430 \mathrm{~nm}$. As it was shown in Fig. 2a, upon increasing the observation wavelength the intensity of the short-wavelength band at $360 \mathrm{~nm}$ decreases. This behavior is accompanied by an increase of the long-wavelength band centered at $430 \mathrm{~nm}$. It is important to note here that each of the area under the fluorescence excitation and fluorescence (Fig. 2a and b) curves were normalized to unity. In this case, each of fluorescence spectrum corresponds to the emission of the same number of emitting EAADCy molecules.

We also carefully examined the excitation-wavelength dependence of fluorescence spectra of EAADCy in THF. We found that upon increasing the excitation wavelength the intensity of short-wavelength fluorescence band decreases. This behavior is accompanied by an increase of the long-wavelength emission band. 


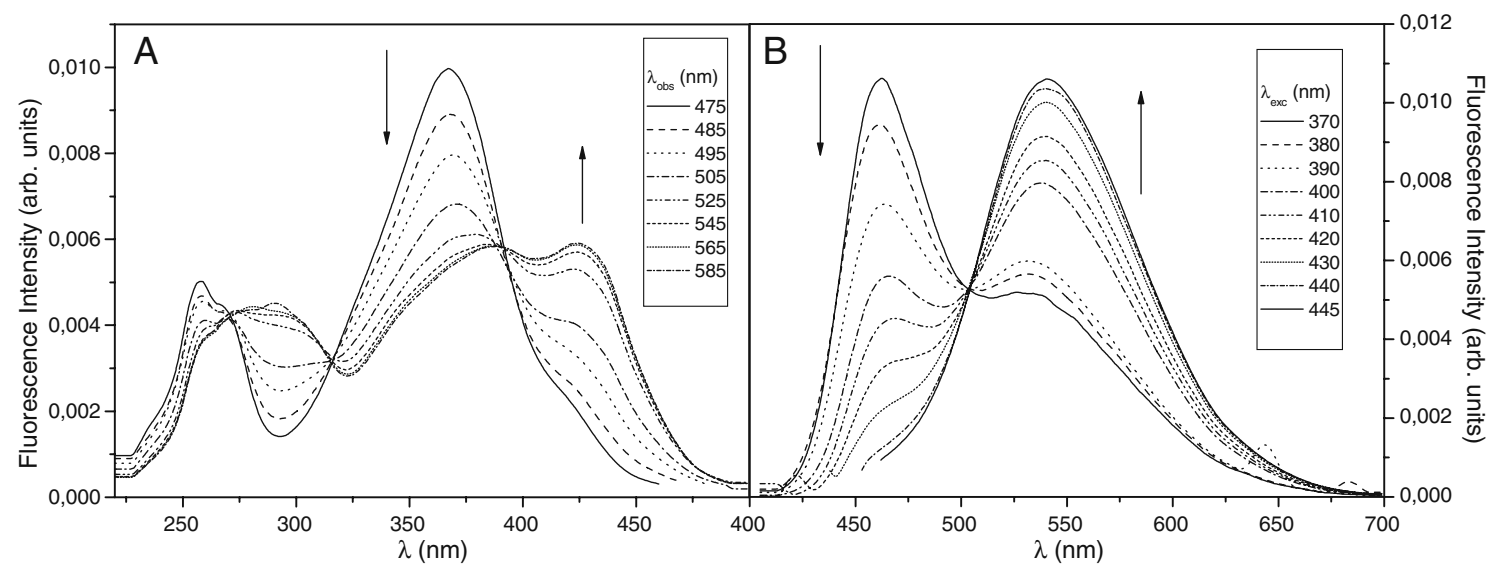

Fig. 2 Fluorescence excitation spectra (a) and fluorescence spectra of EAADCy in THF at room temperature as a function of $\lambda_{\text {obs }}(\mathbf{a})$ and $\lambda_{\text {exc }}(\mathbf{b})$

Analyzing the fluorescence excitation (Fig. 2a) and fluorescence (Fig. 2b) spectra of EAADCy in THF obtained at different observation and excitation wavelengths, it is found that the ratio of the fluorescence intensity of the long-wavelength and short-wavelength fluorescence bands $\left(I_{e m}(L W) / I_{e m}(S W)\right)$ strongly depend on the excitation wavelength. A typical excitation- and observationwavelength dependent behavior is highlighted in Fig. 3. As it can be seen (Fig. 3b), when the excitation wavelength is increased from $360 \mathrm{~nm}$ to $445 \mathrm{~nm}$, the ratio $I_{e m}(L W) / I_{e m}$ $(S W)$ increases from $\sim 0.5$ to $\sim 8$. It is important to note here that the ratio $I_{e m}(L W) / I_{e m}(S W)$ is of the solute concentration independent (with the limit of experimental error).

The steady-state fluorescence excitation spectra are also strongly observation-wavelength dependent. As can be seen (Fig. 3a) the ratio $I_{\text {exc }}(S W) / I_{\text {exc }}(L W)$ undergoes a significant decrease when increasing the observation wavelength (e.g., from $\sim 6.5\left(\lambda_{o b s}=480 \mathrm{~nm}\right)$ to $\sim 0.8\left(\lambda_{o b s}=605 \mathrm{~nm}\right)$. This observation/excitation wavelength dependence is similar to the typical red-edge effect observed in low-temperature glasses, polymer matrixes, and organized assemblies such as micelles, vesicles, proteins and membranes [2-15].

These results, as well as, our previous study [10, 18-20] indicate that for EAADCy a distribution of spatial conformers (lie in difference of rotational angles between donor and acceptor moieties) is always present (see Fig. 1). When the excitation is carried out at the maximum of the absorption band, the conformers having perpendicular or nearly perpendicular geometry $\left(\varphi \sim 90^{\circ}\right)$ are excited. As the excitation wavelength is shifted to the red, the rotamer with different angles $\left(0<\varphi<90^{\circ}\right)$ between their interatomic planes is selectively excited. It can be concluded that $I C T$ state (long-wavelength fluorescence band) may be formed either by further geometrical changes of the normal conformer $\left(\varphi \sim 90^{\circ}\right)$ in the locally excited state or directly by excitation of flattened conformers.
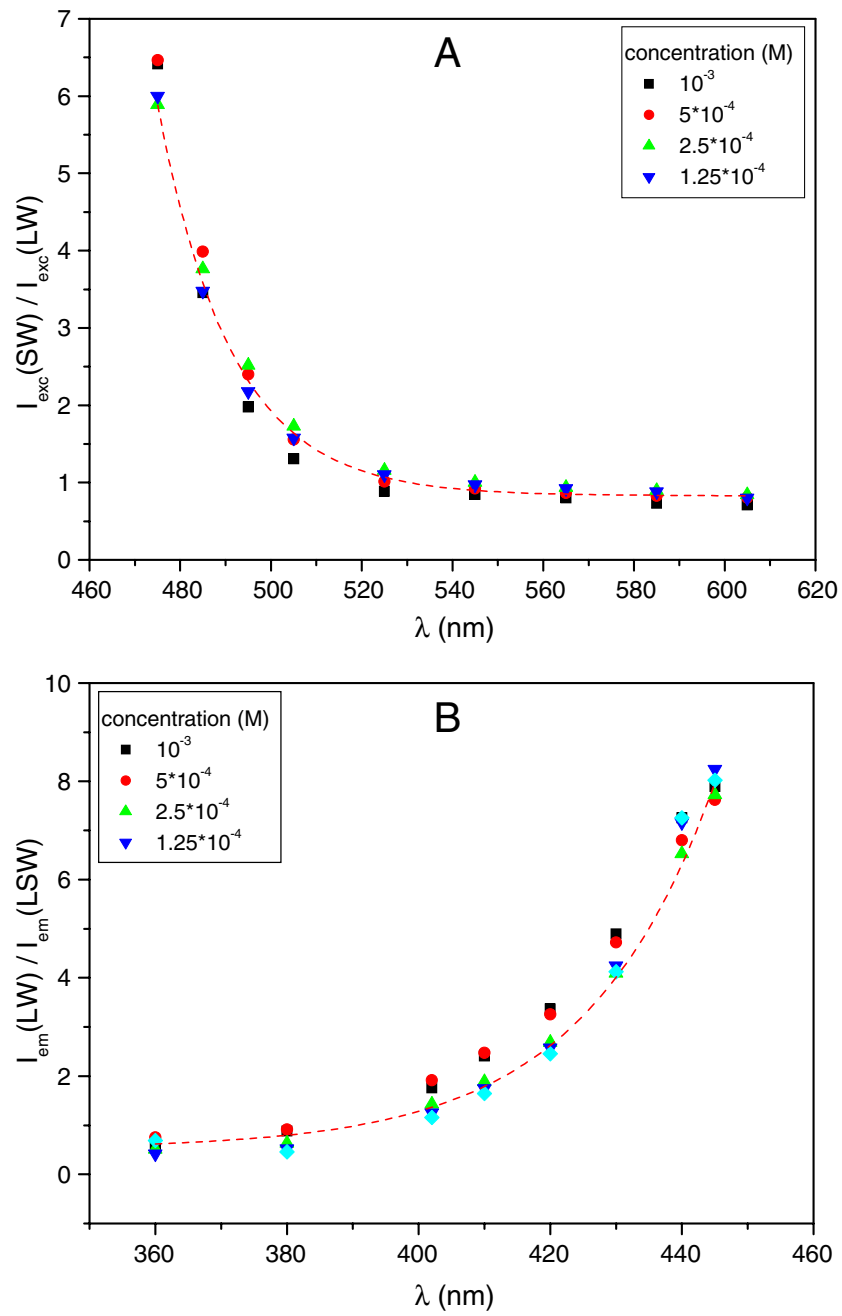

Fig. 3 The ratio $I_{e x c}(\mathrm{SW}) / I_{e x c}(L W)$ (a) and $I_{e m}(L W) / I_{e m}(S W)(\mathbf{b})$ as a function of $\lambda_{o b s}$ and $\lambda_{\text {exc }}$, respectively 
Time-resolved Emission Spectra and Fluorescence Decay Times of EAADCy

In order to confirm the interpretation of the steady-state measurements data, the time-resolved emission spectra and from them calculated fluorescence decay times of EAADCy in THF were investigated. These measurements were carried out at $5 \mathrm{~ns}$ range of streak camera window, what gave us point-to point time resolution of 10.86 ps per point. Figure 4 shows the emission spectra of EAADCy in THF at various times after excitation $\left(\lambda_{e x c}=400 \mathrm{~nm}\right)$. As can be seen, upon increasing the time after excitation the short-wavelength $(L E)$ and longwavelength (ICT) fluorescence maxima positions are redshifted. Figure 4 clearly shows that a pronounced shift of the SW and LW bands occurs already at very short time after excitation. In order to make it more evident, the temporal behavior of $\widetilde{v}_{S W}^{\max }(t)$ and $\widetilde{v}_{L W}^{\max }(t)$ are shown in Fig. 5.

In order to determine the Stokes-shift correlation function $c$ $(t)$, the time-dependent frequencies $\widetilde{v}_{S W}^{\max }(t)$ and $\widetilde{v}_{S W}^{\max }(t)$ are normalized according to Eq. 4. Figure 6 illustrates the resulting $c(t)$ curves for short-wavelength and longwavelength fluorescence bands. The decay times (obtained for different $\lambda_{\text {exc }}$ ) of $c(t)$ are summarized in Table 1 . Analyzing the experimental decay data, it follows that the obtained $\tau_{S W}^{L}$ and $\tau_{L W}^{L}$ values do not depend on $\lambda_{\text {exc. }}$. From the $\tau_{S W}^{L}$ and $\tau_{L W}^{L}$ values obtained for both of the conformers ("perpendicular" $-L E$ form, "flattened"- $I C T$ form), it is evident that the longitudinal relaxation time is very different. The ratio of the corresponding longitudinal relaxation times $\frac{\tau_{S W}^{L}}{\tau^{L}}$ is about 3 . This large difference between $\tau_{S W}^{L}$ and $\tau_{L W}^{L}$ values is understandable taking into account different interactions of the conformers with the reactive electric field of the surrounding solvent. In our previous publication [18], it was shown that the "flattened" (ICT) form of EAADCy possesses greater dipole moment in the excited state than in the ground state, while "perpendicular" $(L E)$ form of EAADCy possesses lower dipole moment value in the

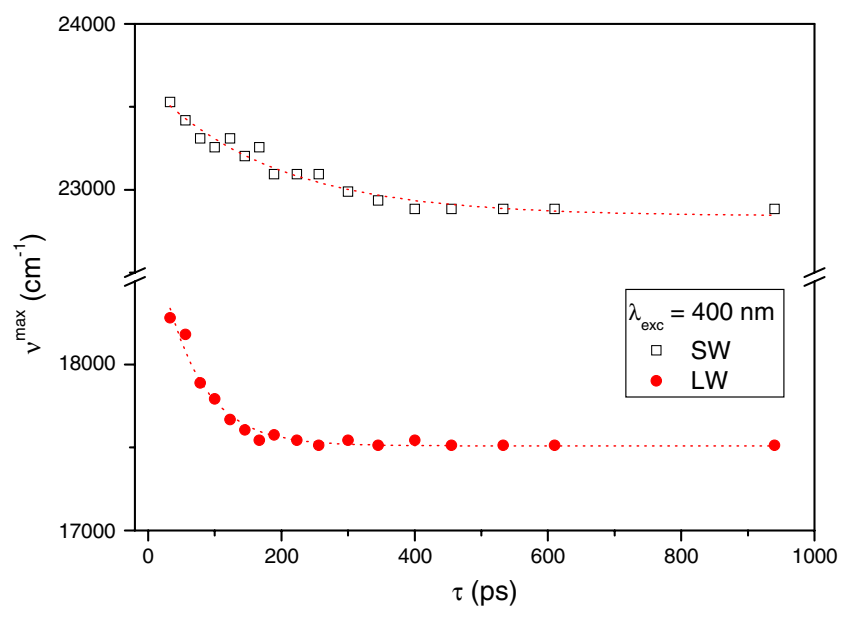

Fig. 5 Time-dependence of the frequency of the short-wavelength (SW) and long-wavelength (LW) emission band maximum of EAADCy in THF

excited state in comparison to its value in the ground state. The following changes of dipole moment were obtained: $\Delta \mu_{\text {perpen. }}=-1.82 \mathrm{D}, \Delta \mu_{\text {flatt. }}=13.5 \mathrm{D}$.

The experimental decay curves were fitted to a multiexponential function $F(t)=\sum_{i=1}^{n} A_{i} \exp (-t / \tau)$, where $A_{i}$ and $\tau_{\mathrm{i}}$ are the preexponential coefficient (describing the contribution of the $i$-th fluorescence decay component to the total emission) and decay time of the $i$-th component, respectively. The average value of $\chi^{2}$, describing the goodness of the fitting procedure for all decay curves, i.e., one- and two-exponential fitting, equals $\chi^{2}=1.2 \pm 0.1$.

Table 1 collects the calculated fluorescence decay time data, $\tau$, and the preexponential factors, $A$, describing the contribution of the $i$-th fluorescence decay component of the total emission. This results point that the luminescent solution of EAADCy in THF established a spectroscopic inhomogeneous system attributed to the emission from locally excited, $S_{1}(L E)$, and intramolecular charge transfer, $S_{1}(I C T)$, states. For all studied excitation wave-
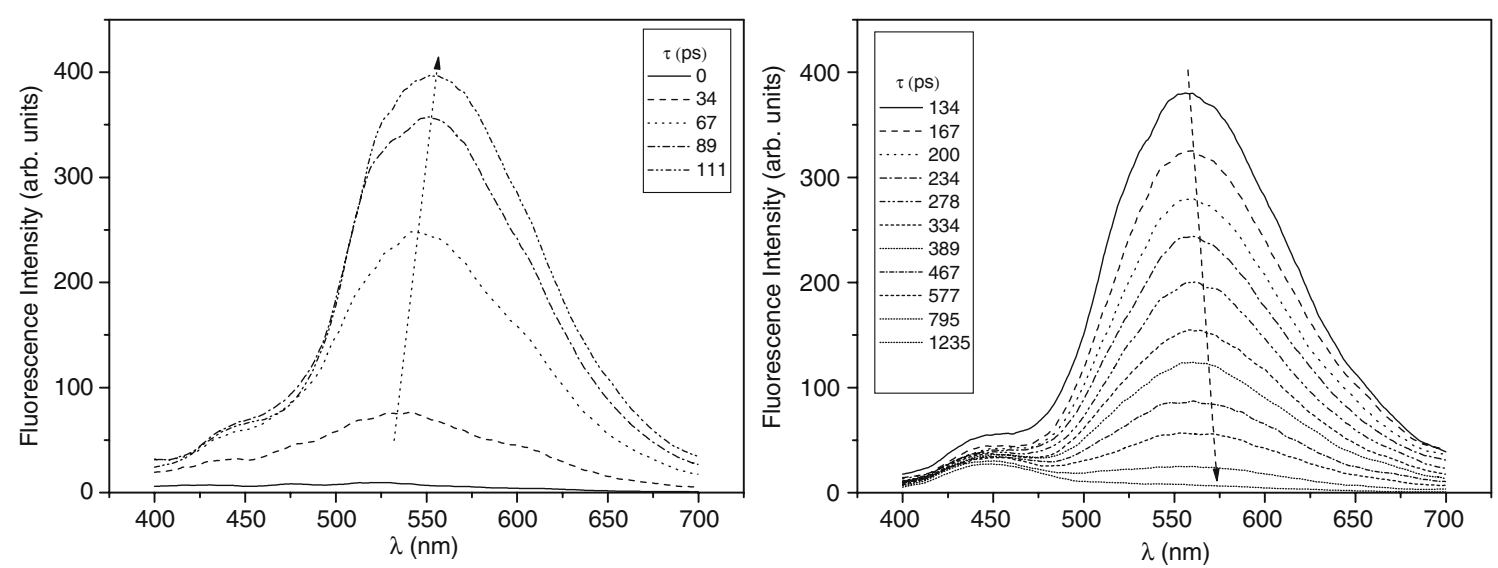

Fig. 4 Time-resolved emission spectra of EAADCy in THF at room temperature at various times after excitation $\left(\lambda_{\text {exc }}=400 \mathrm{~nm}\right)$ 

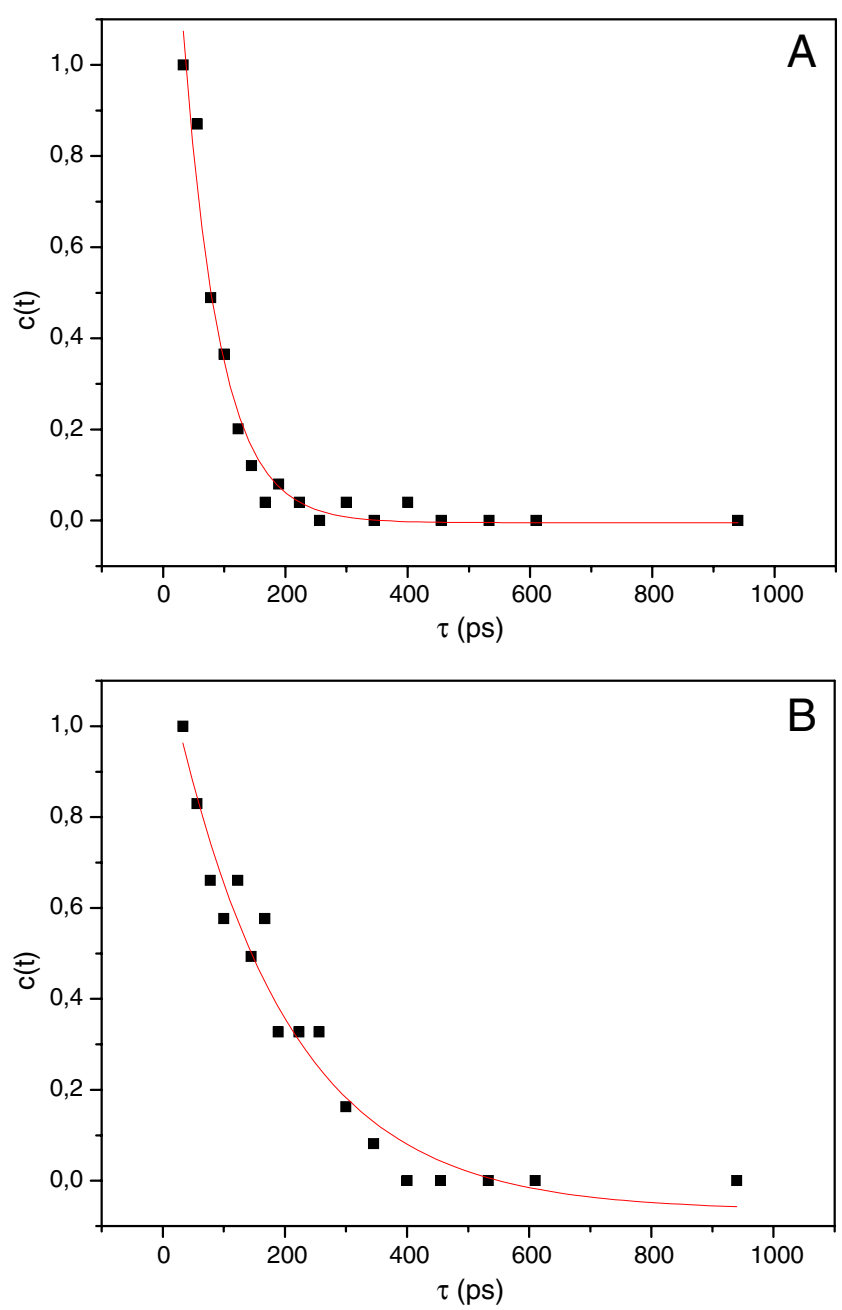

Fig. 6 Decay of the solvent correlation function $c(t)$ of LE (b) and ICT (a) form of EAADCy in THF at room temperature

lengths $(370 \mathrm{~nm}, 390 \mathrm{~nm}, 400 \mathrm{~nm}, 420 \mathrm{~nm})$ emission decay exhibits fast $\left(\tau_{L W} \cong 0.22 n s\right)$ and slow $\left(\tau_{S W} \cong 5.7 n s\right)$ decay components. The slow decay component originated from the emission of locally excited state (perpendicular conformer), whereas the fast decay component results from an ensemble of spatial conformers possessing the
$I C T$ structure (flattened conformer). It is important to note here that the relative amplitude of $L E$ and ICT emissions strongly depend on the excitation wavelength. The preexponential factor $A_{S W}$ (describing the contribution of the $L E$ fluorescence decay component of the total emission) decreases with increasing excitation wavelength from $78 \%$ for excitation at $370 \mathrm{~nm}$ to $43 \%$ for excitation at $420 \mathrm{~nm}$. In contrast, as the excitation wavelength is increased from $370 \mathrm{~nm}$ to $420 \mathrm{~nm}$, the $A_{L W}$ increases from $22 \%$ to $57 \%$.

\section{Conclusions}

Analyzing the data obtained from the steady-state, timeresolved measurements and quantum-chemical calculations one can state that:

- At room temperature, EAADCy exists as an equilibrium mixture of various conformers having different geometry (in the range from $60^{\circ}$ to $120^{\circ}$ ) and absorb at different wavelengths. As the excitation wavelength is changed, a slightly different species are excited.

- The ratio of the fluorescence intensity of the longwavelength and short-wavelength fluorescence bands strongly depends on the observation/excitation wavelength, whereas the wavelengths of the fluorescence excitation and fluorescence bands maxima are independent on the observation/excitation wavelengths in accordance to the Kasha rule.

- The difference between Stokes shift in the $L E$ and ICT states was attributed to the solvent response to the large photoinduced dipole moment of EAADCy in the fluorescent charge transfer state.

- The excitation wavelength dependent biexponential decay times (and preexponential factors $A_{S W}$ and $A_{L W}$ ) of EAADCy in THF lead us to conclude that $I C T$ state really may be formed either by further geometrical changes of the locally excited conformer or directly by excitation of flattened conformers.
Table 1 The longitudinal relaxation times $\left(\tau_{S W}^{L}, \tau_{L W}^{L}\right)$, fluorescence decay times $\left(\tau_{S W}, \tau_{L W}\right)$ and preexponential factors $\left(A_{S W}, A_{L W}\right)$ of EAADCy in THF

\begin{tabular}{|c|c|c|c|c|c|c|}
\hline$\tau_{S W}^{L}(\mathrm{ps})$ & $\tau_{L W}^{L}(\mathrm{ps})$ & $\tau_{S W}(\mathrm{ps})$ & $A_{S W}(\%)$ & $\tau_{L W}(\mathrm{ps})$ & $A_{L W}(\%)$ & $\chi^{2}$ \\
\hline & & $\lambda_{e x c}=370 \mathrm{~nm}$ & & & & \\
\hline \multirow[t]{2}{*}{$162 \pm 20$} & $53 \pm 4$ & 5.65 & 78.2 & 0.22 & 21.8 & 1.21 \\
\hline & & $\lambda_{\text {exc }}=390 \mathrm{~nm}$ & & & & \\
\hline \multirow[t]{2}{*}{$170 \pm 15$} & $64 \pm 8$ & 5.83 & 66.4 & 0.21 & 33.6 & 1.09 \\
\hline & & $\lambda_{\text {exc }}=400 \mathrm{~nm}$ & & & & \\
\hline \multirow[t]{2}{*}{$187 \pm 20$} & $59 \pm 5$ & 5.73 & 53.3 & 0.25 & 46.7 & 1.18 \\
\hline & & $\lambda_{\text {exc }}=420 \mathrm{~nm}$ & & & & \\
\hline $182 \pm 12$ & $62 \pm 8$ & 5.55 & 43.2 & 0.20 & 56.8 & 1.22 \\
\hline
\end{tabular}


Acknowledgements The authors express their gratitude to Professor Józef Heldt, and Professor Jerzy Pączkowski for scientific discussions and valuable comments on the manuscript. This work was supported by The Ministry of Science and Higher Education of Poland research Grant NN204 054335 and by The University of Gdansk, Grant BW5209-5-0348-0. The authors are grateful for these supports.

Open Access This article is distributed under the terms of the Creative Commons Attribution Noncommercial License which permits any noncommercial use, distribution, and reproduction in any medium, provided the original author(s) and source are credited.

\section{References}

1. Lakowicz JR (2006) Principle of fluorescence spectroscopy. Kluwer Academic/Plenum Publishing Corporation, New York

2. Demchenko AP (2002) Luminescence 17:19

3. Demchenko AP, Sytnik AI (1991) Proc Natl Acad Sci USA 88:9311

4. Sengupta B, Guharay J, Chakraborty A, Sengupta PK (2002) Spectrochim Acta A 58:2005

5. Kallir AJ, Suter GW, Wild UP (1987) J Phys Chem 91:60

6. Demchenko AP, Sytnik AS (1991) J Phys Chem 95:10518
7. Nemkovich NA, Rubinov AN, Tomin VI (1991) Inhomogeneous broadening of electronic spectra of dye molecules in solutions, Chap. 8. In: Lakowicz JR (ed) Topics in fluorescence spectroscopy. Plenum, New York

8. Chattopadhyay A, Mukherjee S (1999) J Phys Chem B 103:8180

9. Józefowicz M, Heldt JR, Heldt J (2002) Z Naturforsch 57 a:787

10. Józefowicz M, Heldt JR, Bajorek A, Pączkowski J (2008) J Photochem Photobiol A 196:38

11. Nemkovich NA, Rubinov AN (1995) J Fluoresc 5:285

12. Nemkovich NA, Kruchenok JV, Rubinov AN, Pivovarenko VG, Baumann W (2001) J Photochem Photobiol A 139:53

13. Guha S, Rawat SS, Chattopadhyay A, Bhattacharyya B (1996) Biochemistry 35:13426

14. Hof M, Lianos P, Laschewsky A (1997) Langmuir 13:2181

15. Hof M, Lianos P (1997) Langmuir 13:290

16. Maroncelli M, Fleming GR (1987) J Chem Phys 86:6221

17. Kubicki A, Bojarski P, Grinberg M, Sadownik M, Kukliński B (2006) Opt Comm 263:275

18. Józefowicz M, Aleksejew M, Heldt JR, Bajorek A, Pączkowski J, Heldt J (2007) Chem Phys 338:53

19. Józefowicz M, Heldt JR, Bajorek A, Pączkowski J (2009) Chem Phys 363:88

20. Józefowicz M, Heldt J, Heldt JR, Aleksiejew M, Iwulska AM, Pączkowski J (2009) Optical Materials 31:1894 